\title{
Primary Pelvic Hydatid Cyst: A Rare Case Report
}

\author{
Authors \\ Dr (Mrs) D.S.Nirhale, Dr Mansi Dhende, Dr Pravin Shingade, Dr Tejas Sonawane \\ Dr Gaurav Kulkarni, Dr Nishant Priyadarshi \\ Department of Surgery, DY Patil University Pimpri Pune India
}

\begin{abstract}
Hydatid disease is caused by a cyclo-zoonotic parasite echinococus granulosus. Humans are accidental intermediate hosts of echinococus. Commonly involved organ is the liver followed by lungs, kidney and musculoskeletal system. Pelvic hydatid cyst is rarely reported. Imaging modalities such as ultrasonography, computed tomography (CT), and magnetic resonance imaging (MRI) are helpful in the pre-operative diagnosis. Serology, may also be helpful in the diagnosis. However a high index of suspicion is required in a case of pelvic hydatid cyst. Here we present a case of primary pelvic hydatid cyst treated by laproscopic excision.

Keywords-hydatid disease, pelvic hydatidcyst,unusual,laproscopic excision.
\end{abstract}

\section{Introduction}

Echinococcosis or hydatid disease is a common zoonotic parasitic disease caused by echinococcus granulosus in tropical countries ${ }^{1}$. Humans are accidental intermediate host and become infected when they accidently ingest eggs of the tapeworm. They are the dead end for the parasite. The disease in humans most commonly occurs in liver followed by the lung, kidney and musculoskeletal system. Other sites such as the heart spleen, pancreas, omentum, ovaries, pelvis or retroperitoneum are rarely affected. We present a rare case of primary pelvic hydatid cyst treated with laproscopic excision.

\section{Case Report}

A 26 years old male patient presented to the surgery OPD with the complaints of pain in the abdomen since 3 months. They were no complaints of abdominal lump, urinary complaints. On examination his pulse was 80/min, BP was $120 / 70 \mathrm{~mm}$ of $\mathrm{Hg}$. Perabdominal examination: tenderness was present in the hypogastric region, no palpable lump.

Patient's blood investigations such as hemogram, LFT and coagulation profile revealed no abnormallities. Echinococus (hydatid serology), IgG too was within normal limits. Abdominal ultrasonography revealed awell defined cystic lesion in the pelvis of size 12.3 (cc)x 10.7(AP)x10.8(T) Posterior to urinary urinary bladder with multiple thick walled cystic lesion noted arranged in a circumferential manner. Patients CECT abdomen and pelvis was suggestive of a round non- enhancing cystic lesion superior to the urinary bladder indenting its dome and mildly compressing both ureters probable benign cyst.

Patient was given pre-operative albendazole theary followed by surgery. Laparoscopy was done which revealed a large cystic mass in the pelvis posterior 


\section{JMSCR Vol||05||Issue||05||Page 21359-21361||May}

to the urinary bladder. Intra operatively Hypertonic saline-soaked pads were used. Pelvic hydatid cyst were removed in to without rupture, and sent for histo-pathological examination the HPE was consistent with the diagnosis of hydatid cyst. Postoperative period was uneventful. Patient has been put on tab albendazole $400 \mathrm{mg}$ twice daily for 6 months.

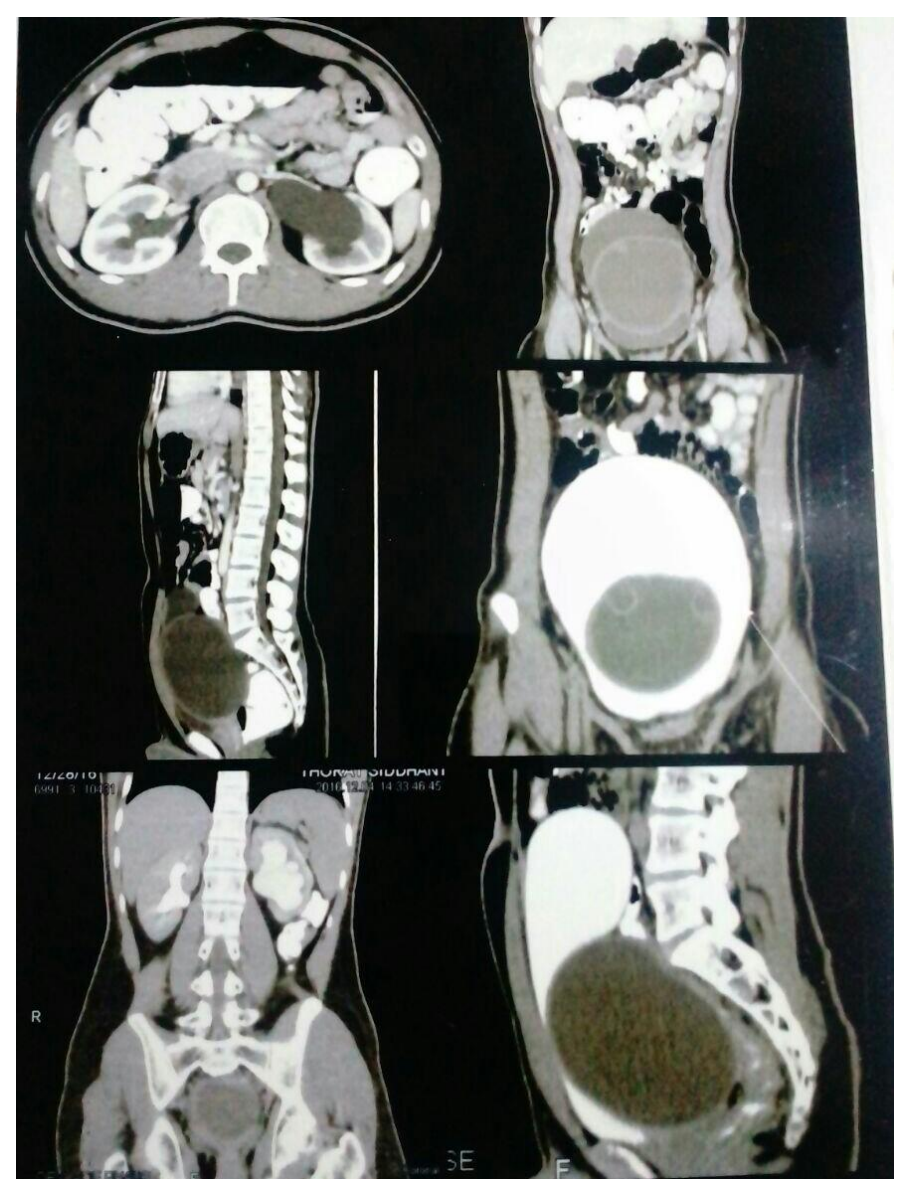

Fig. 1 CT image showing the cyst posterior to the bladder

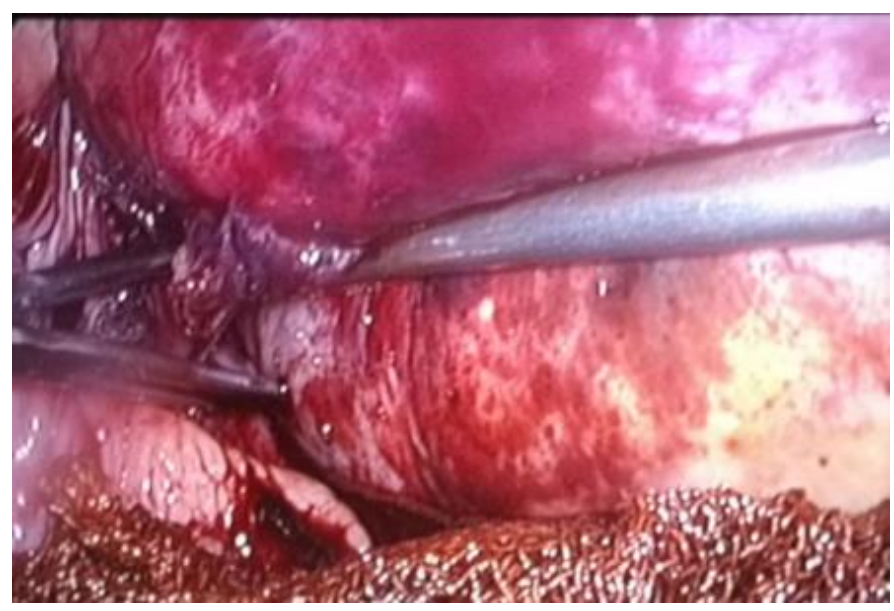

Fig 2 Intra operative image showing the cyst

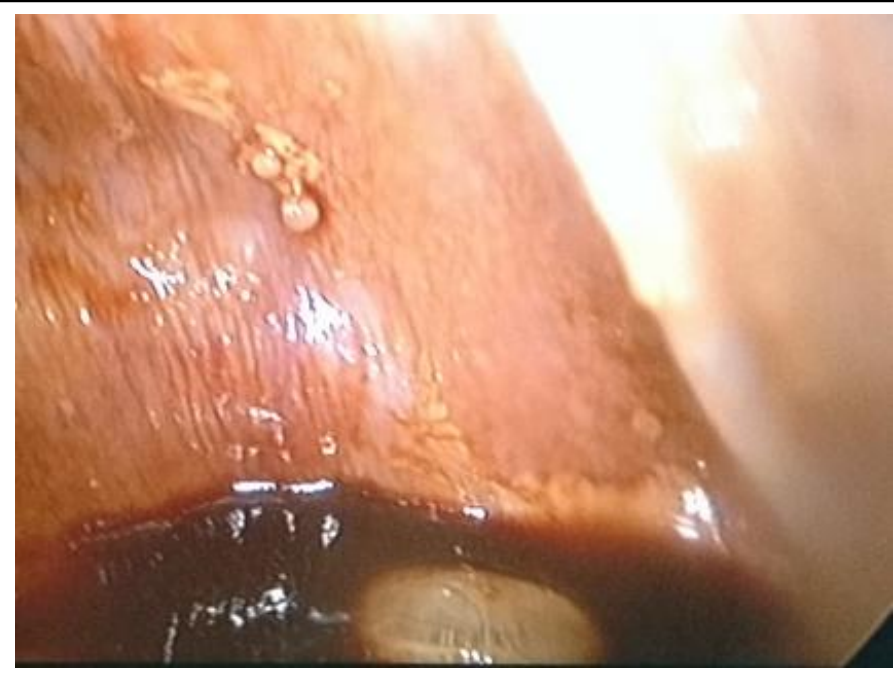

Fig 3. Intra operative image showing the daughter cyst

\section{Discussion}

Hydatid disease is a parasitic disease. Four species known to cause infection in humans are echinococcus granulosum, echinococcus mutilocularis, echinococus vogeli and echinococcus oligarthis ${ }^{2}$. Commonly involved sites in order of frequency are : liver (59-75\%), lungs (27\%), kidney(3\%), bone(1$4 \%)$ and brain (1-2\%) other sites are rarely involved $^{3}$. Peritoneal cavity involvement in hydatid disease is found in 10 to $16 \%$ of cases ${ }^{4}$.

Peritoneal hydatid disease is commonly seen secondary to previous surgery for liver hydatidosis. Release of brood's capsule, scolices and daughter cysts after intra perinoneal rupture of hepatic or splenic cyst results in the development of multiple disseminated intraperitoneal hydatid disease, this phenomenon is called secondary echinococcosis ${ }^{5}$. Primary peritoneal echinococcosis is rare. The mechanism of primary peritoneal infection is unknown ${ }^{6}$.

Diagnosis is most commonly made through USG or CT scan of the abdomen. Daughter cysts and hydatid sand are seen, and there maybe associated wall calcification. CT scan is the imaging modality of choice for peritoneal disease. Several serological tests are also used in the diagnosis. Detection of the antigen is less sensitive than antibody detection. ELISA has a sensitivity ranging from $64 \%$ to $100 \%$. Surgery is the treatment of choice. Combination of preoperative albendazole therapy, surgery, and 
postoperative albendazole therapy is the preferred regime.

\section{Conclusions}

Primary Pelvic hydatid disease though rare should not be missed. The treatment of choice remains preoperative albendazole followed by complete excision of the cyst and post-operative aldendazole for 6 months.

\section{References}

1. Akhtar MJ khanamN, RaoS.Clinical epidemiological profile of hydatid disease in Central India, a retrospective and prospective study.Int j Biol Med Res 2011;2:60-1.

2. M.S.Khuroo" hydatiddisease :current status and recent advances"; Annals of Saudi Medicine, vol 22, pp 56-64,2002.

3. M Yuksel,GDemirpolat,A Sever, S Bakaris, E Bulbouloglu,MElmas.Hydatid disease involving some rare locations in the body: a oictorialessay.The Korean Journal of Radiology, vol. 8,no 6,pp531-540,2007.

4. Prousalidis J, Tzardinoglouk K, Katsokis C, Aletras H. Uncommon sites of hydatid disease. World J Surg. 1998;22:17-22

5. Sahu SK, Singh P, Sachan PK, et al. Secondary echinococcosis. Indian J Surg. 2006;68:334-335

6. Nadeem N, Khan H, Fatimi S, Ahmad MN. Giant multiple intraabdominalhydatid cysts: case report. J Ayub Med Coll Abbottabad. 2006;18:71-3 